\title{
ALMOST SURE VERSIONS OF LIMIT THEOREMS FOR RANDOM SUMS OF MULTIINDEX RANDOM VARIABLES
}

\author{
A.N. Chuprunov, L.P. Terekhova \\ Chebotarev Inst. of Mathematics and Mechanics, Kazan State University \\ Kazan, RUSSIA \\ e-mail: achuprunov@mail.ru; t-lidia@yandex.ru
}

\begin{abstract}
In the case of the domain of attraction of a $p$-stable law almost sure versions of limit theorems for random vectors are presented.
\end{abstract}

\section{Introduction}

We will suppose that $0<p \leq 2$. Let's denote by $\stackrel{d}{\longrightarrow}$ the convergence in distribution, by $\stackrel{w}{\longrightarrow}$ the weak convergence of measures, by $\mu_{\zeta}$ the distribution of the random variable $\zeta$ and by $\mathbf{R}$ the set of real numbers.

Let $\zeta_{n}, n \in \mathbf{N}$, be a sequence of random variables defined on the probability space $(\Omega, \mathcal{A}, \mathbf{P})$. Consider the measures

$$
Q_{n}(\omega)=Q_{n}\left(\left(\zeta_{n}\right)\right)(\omega)=\frac{1}{\log n} \sum_{k=1}^{n} \frac{1}{k} \delta_{\zeta_{k}(\omega)},
$$

where $\omega \in \Omega, n \in \mathbf{N}$ and $\delta_{x}$ is the point mass at $x$.

Classical limit theorems deal with the following convergence: $\zeta_{n} \stackrel{d}{\longrightarrow} \zeta$, as $n \rightarrow \infty$. In many cases the convergence $\zeta_{n} \stackrel{d}{\longrightarrow} \zeta$, as $n \rightarrow \infty$, implies the convergence of measures $Q_{n}(\omega) \stackrel{w}{\longrightarrow} \mu_{\zeta}$, as $n \rightarrow \infty$, for almost all $\omega \in \Omega$. Such limit theorems are called almost sure versions of ordinary limit theorems. Investigations in this field started with Brosambler [3] and Schatte [8], who obtained an almost sure version of the central limit theorem. Then Berkes I. [1] and Ibragimov I.A. [5] generalized their results on the normalized sums of identically distributed random variables that belong to the domain of attraction of a $p$-stable law. Berkes I. and Csáki E. [2] showed that every weak limit theorem for random variables, subject to minor technical conditions, has an analogous almost sure version. Also a paper of Fazekas I. and Rychlik Z. [4] should be noted. There an almost sure version of the central limit theorem for the sums of multiindex random variables is presented.

Let $\xi, \xi_{n}, n \in \mathbf{N}$, be independent identically distributed random variables defined on the probability space $(\Omega, \mathcal{A}, \mathbf{P})$, which belong to the domain of attraction of a $p$ stable law. It means that for some numerical sequence $B_{n}$, such that $B_{n} \longrightarrow \infty$, as $n \rightarrow \infty$, the following convergence takes place:

$$
S_{n} \stackrel{d}{\longrightarrow} \gamma_{p}, n \rightarrow \infty
$$


where $S_{n}=\frac{1}{B_{n}} \sum_{i=1}^{n}\left(\xi_{i}-\alpha_{n}\right), \alpha_{n}=E \xi_{1} \cdot I_{\left|\frac{\xi_{1}}{B_{n}}\right|<1}$ and $\gamma_{p}$ is a $p$-stable random variable.

An almost sure version of the limit theorem (1) was obtained by Ibragimov I.A. in [5] and Berkes I. in [1]. Let's consider the sums of random variables with a random index of summation

$$
S_{n}^{\nu}=\frac{1}{B_{n}} \sum_{i=1}^{\nu_{n}}\left(\xi_{i}-\alpha_{n}\right)=\frac{1}{B_{n}} \sum_{i=1}^{\infty}\left(\sum_{k=1}^{i}\left(\xi_{k}-\alpha_{n}\right)\right) \cdot I_{\left\{\nu_{n}=i\right\}}
$$

where $\nu_{n}, n \in \mathbf{N}$, is a sequence of integer-valued random variables, defined on the probability space $(\Omega, \mathcal{A}, \mathbf{P})$.

Renyi in [7] investigated the convergence in distribution of the sequence in (2).

Consider the sequence of random vectors $V_{n}=\left(S_{n}, W_{n}\right), n \in \mathbf{N}$, where $W_{n}=$ $\frac{1}{B_{n}^{2}} \sum_{i=1}^{n}\left(\xi_{i}-\alpha_{n}\right)^{2}$. The following limit theorem is an isolated case of the theorem, obtained by Kruglov V. M. and Petrovskaya G.N. [6].

Theorem A Let $S_{n} \stackrel{d}{\longrightarrow} \gamma_{p}$, as $n \rightarrow \infty$, where $\gamma_{p}$ is a $p$-stable random variable with the characteristic function

$f(t)=\exp \left\{\int_{-\infty}^{0}\left(e^{i t x}-1-i t \frac{x}{1+x^{2}}\right) d\left(\frac{c_{1}}{|x|^{p}}\right)+\int_{0}^{\infty}\left(e^{i t x}-1-i t \frac{x}{1+x^{2}}\right) d\left(-\frac{c_{2}}{x^{p}}\right)\right\}$,

where $t \in \mathbf{R}, c_{1}, c_{2} \geq 0, c_{1}+c_{2}>0$.

Then the sequence of distribution functions of random vectors $\left(S_{n}, W_{n}\right), n \in \mathbf{N}$, weakly converges to the distribution function with the characteristic function

$$
\begin{aligned}
f(s, t) & =\exp \left\{\int_{-\infty}^{0}\left(e^{i s x+i t x^{2}}-1-i s \frac{x}{1+x^{2}}\right) d\left(\frac{c_{1}}{|x|^{p}}\right)+\right. \\
& \left.+\int_{0}^{\infty}\left(e^{i s x+i t x^{2}}-1-i s \frac{x}{1+x^{2}}\right) d\left(-\frac{c_{2}}{x^{p}}\right)\right\}
\end{aligned}
$$

where $s, t \in \mathbf{R}, c_{1}, c_{2} \geq 0, c_{1}+c_{2}>0$.

Let $\mathbf{k}=\left(k_{1}, k_{2}, \ldots, k_{d}\right), \mathbf{n}=\left(n_{1}, n_{2}, \ldots, n_{d}\right), \ldots \in \mathbf{N}^{d},|\mathbf{n}|=\prod_{i=1}^{d} n_{i}$ and $|\log \mathbf{n}|=$ $\prod_{i=1}^{d} \log _{+} n_{i}, \mathbf{n} \in \mathbf{N}^{d}$, where $\log _{+} x=\log x$, if $x \geq e$, and $\log _{+} x=1$, if $x<e$.

Let $\zeta_{\mathbf{n}}, \mathbf{n} \in \mathbf{N}^{d}$, be a sequence of random variables defined on the probability space $(\Omega, \mathcal{A}, \mathbf{P})$. Consider the measures $Q_{\mathbf{n}}(\omega)=Q_{\mathbf{n}}\left(\left(\zeta_{n}\right)\right)(\omega)=\frac{1}{|\log \mathbf{n}|} \sum_{\mathbf{k} \leq \mathbf{n}} \frac{1}{|\mathbf{k}|} \delta_{\zeta_{\mathbf{k}}(\omega)}$. The multiindex version of the ordinary almost sure limit theorem is the following:

$$
Q_{\mathbf{n}}\left(\left(\zeta_{n}\right)\right)(\omega) \stackrel{w}{\longrightarrow} \mu_{\zeta}, n \rightarrow \infty
$$

for almost all $\omega \in \Omega$.

Let $\xi_{\mathbf{k}}, \mathbf{k} \in \mathbf{N}^{d}$, be the multiindex sequence of independent identically distributed random variables, which belong to the domain of attraction of a $p$-stable law. Theorem A remains valid in the case of the multiindex sequences $V_{\mathbf{n}}=\left(S_{\mathbf{n}}, W_{\mathbf{n}}\right), \mathbf{n} \in \mathbf{N}^{d}$, where

$$
S_{\mathbf{n}}=\frac{1}{B_{|\mathbf{n}|}} \sum_{\mathbf{i} \leq \mathbf{n}}\left(\xi_{\mathbf{i}}-\alpha_{|\mathbf{n}|}\right), \quad W_{\mathbf{n}}=\frac{1}{B_{|\mathbf{n}|}^{2}} \sum_{\mathbf{i} \leq \mathbf{n}}\left(\xi_{\mathbf{i}}-\alpha_{|\mathbf{n}|}\right)^{2},
$$


$\alpha_{|\mathbf{n}|}=E \xi_{1} I_{\left|\frac{\xi_{1}}{B|\mathbf{n}|}\right|<1}, B_{|\mathbf{n}|}$ is a numerical sequence, such that $B_{|\mathbf{n}|} \rightarrow \infty$, as $\mathbf{n} \rightarrow \infty$, and the convergence $S_{\mathbf{n}} \stackrel{d}{\longrightarrow} \gamma_{p}$, as $\mathbf{n} \rightarrow \infty$, takes place.

Let $\nu_{\mathbf{n}}=\left(\nu_{1 \mathbf{n}}, \nu_{2 \mathbf{n}}, \ldots, \nu_{d \mathbf{n}}\right)$, where $\nu_{1 \mathbf{n}}, \nu_{2 \mathbf{n}}, \ldots, \nu_{d \mathbf{n}}: \Omega \longrightarrow \mathbf{N}$, be sequences of integervalued random vectors.

Our aim is to generalize Theorem A to the case of 2-dimensional random vectors $\left(S_{\mathbf{n}}^{\nu}, W_{\mathbf{n}}^{\nu}\right)$ with the coordinates $S_{\mathbf{n}}^{\nu}=\frac{1}{B_{|\mathbf{n}|}} \sum_{\mathbf{i} \leq \nu_{\mathbf{n}}}\left(\xi_{\mathbf{i}}-\alpha_{|\mathbf{n}|}\right)$ and $W_{\mathbf{n}}^{\nu}=\frac{1}{B_{|\mathbf{n}|}^{2}} \sum_{\mathbf{i} \leq \nu_{\mathbf{n}}}\left(\xi_{\mathbf{i}}-\right.$ $\left.\alpha_{|\mathbf{n}|}\right)^{2}$, and to get an almost sure version of this result.

\section{Main results}

Below we formulate our first result providing the convergence in distribution of random vectors $V_{\mathbf{n}}^{\nu}=\left(S_{\mathbf{n}}^{\nu}, W_{\mathbf{n}}^{\nu}\right)$.

Theorem 1. Assume that $\left(\frac{\nu_{1 \mathbf{n}}}{n_{1}}, \frac{\nu_{2 \mathbf{n}}}{n_{2}}, \ldots, \frac{\nu_{d \mathbf{n}}}{n_{d}}\right) \stackrel{d}{\longrightarrow}\left(\nu_{1}, \nu_{2}, \ldots, \nu_{d}\right)$, as $\mathbf{n} \rightarrow \infty, \mathbf{n} \in \mathbf{N}^{d}$, $\left\{\nu_{\mathbf{n}}\right\}$ and $\left\{\xi_{\mathbf{n}}\right\}$ are independent. Let $S_{\mathbf{n}} \stackrel{d}{\longrightarrow} \gamma_{p}$, as $\mathbf{n} \rightarrow \infty$, where $\gamma_{p}$ is a p-stable random variable.

Then $V_{\mathbf{n}}^{\nu} \stackrel{d}{\longrightarrow} V^{\nu}$, as $\mathbf{n} \rightarrow \infty$, where $V^{\nu}$ is a random vector with the characteristic function

$$
f^{\nu}(s, t)=\int_{0}^{\infty} \int_{0}^{\infty} \ldots \int_{0}^{\infty} f^{|\mathbf{u}|}(s, t) d \mu_{\nu_{1}}\left(u_{1}\right) d \mu_{\nu_{2}}\left(u_{2}\right) \ldots d \mu_{\nu_{d}}\left(u_{d}\right)
$$

and $f(s, t)$ is defined by (3).

The following theorem is an almost sure version of Theorem 1.

Theorem 2. Assume that $\nu_{\mathbf{n}}$ is a sequence of independent random variables, $\left\{\nu_{\mathbf{n}}\right\}$ and $\left\{\xi_{\mathbf{n}}\right\}$ are independent, $\left(\frac{\nu_{1 \mathbf{n}}}{n_{1}}, \frac{\nu_{2 \mathbf{n}}}{n_{2}}, \ldots, \frac{\nu_{d \mathbf{n}}}{n_{d}}\right) \stackrel{d}{\longrightarrow}\left(\nu_{1}, \nu_{2}, \ldots, \nu_{d}\right)$, as $\mathbf{n} \rightarrow \infty$. Let $S_{\mathbf{n}} \stackrel{d}{\longrightarrow} \gamma_{p}$, as $\mathbf{n} \rightarrow \infty$, where $\gamma_{p}$ is a $p$-stable random variable.

Then for almost all $\omega \in \Omega$ it holds that

$$
Q_{\mathbf{n}}\left(\left(V_{\mathbf{n}}^{\nu}\right)\right)(\omega) \stackrel{w}{\longrightarrow} \mu_{V^{\nu}}, \mathbf{n} \rightarrow \infty .
$$

Corollary 1. Assume that the conditions of Theorem 2 are valid.

(a) Let $Q_{\mathbf{n}}(\omega)=Q_{\mathbf{n}}\left(\left(S_{\mathbf{n}}^{\nu}\right)\right)(\omega)$. Then for almost all $\omega \in \Omega$ we have

$$
Q_{\mathbf{n}}\left(\left(S_{\mathbf{n}}^{\nu}\right)\right)(\omega) \stackrel{w}{\longrightarrow} \mu_{\gamma_{p}^{\nu, 1}}, \mathbf{n} \rightarrow \infty .
$$

(b) Let $Q_{\mathbf{n}}(\omega)=Q_{\mathbf{n}}\left(\left(S_{\mathbf{n}}^{\nu}\right)\right)(\omega)$. Then for almost all $\omega \in \Omega$ we have

$$
Q_{\mathbf{n}}\left(\left(W_{\mathbf{n}}^{\nu}\right)\right)(\omega) \stackrel{w}{\longrightarrow} \mu_{\gamma_{p}^{\nu, 2}}, \mathbf{n} \rightarrow \infty,
$$

where $\gamma_{p}^{\nu, 1}$ and $\gamma_{p}^{\nu, 2}$ are coordinates of the random vector $V^{\nu}$ from Theorem 2, which are defined by projections of the characteristic function (4) on the first and the second coordinate, respectively, i.e. by characteristic functions $f(s, 0)$ and $f(0, t)$. 
now we will give an almost sure version of multiindex limit theorem for Student's statistics.

A function $h: \mathbf{R} \times \mathbf{R}_{+} \longrightarrow \mathbf{R}$ is denoted in the following way. Let $h(x, y)=x / \sqrt{y}$ for $y \neq 0$, and $h(x, y)=0$ for $y=0$. Then we consider the sequence of self-normalized sums $T_{\mathbf{n}}^{\nu}=h\left(S_{\mathbf{n}}^{\nu}, W_{\mathbf{n}}^{\nu}\right)$.

Theorem 3. Let $\nu(\{0\})=0, Q_{\mathbf{n}}(\omega)=Q_{\mathbf{n}}\left(\left(T_{\mathbf{n}}^{\nu}\right)\right)(\omega)$. Then under the conditions of Theorem 2 for almost all $\omega \in \Omega$ we have

$$
Q_{\mathbf{n}}\left(\left(T_{\mathbf{n}}^{\nu}\right)\right)(\omega) \stackrel{w}{\longrightarrow} \mu_{T^{\nu}}, \mathbf{n} \rightarrow \infty
$$

where $\mu_{T^{\nu}}$ is an image of the measure $\mu_{V^{\nu}}$ according to the mapping $h$.

We note that instead of the condition of independency of $\nu_{\mathbf{n}}$ in Theorem 2 it is possible to use the following condition: $\nu_{\mathbf{l}}$ and $\nu_{\mathbf{l n}}, \mathbf{l}<\mathbf{n}$, are independent, and $E \nu_{\mathbf{n}} \leq$ $C \mathbf{n}$, where $C>0$ is some constant, and $\nu_{\mathbf{n}}=\nu_{\mathbf{l}}+\nu_{\mathbf{l n}}$.

Also random sums almost sure limit theorems for the domain of geometric partial attraction of a semistable law and almost sure versions of limit theorems for geometric random sums have been obtained.

\section{References}

[1] Berkes I. (1995). On the almost sure central limit theorem and domains of attraction. Probab. Theory Related Fields. Vol. 102, pp. 1-18.

[2] Berkes I., Csáki E. (2001). A universal result in almost sure central limit theorem. Stoch. Proc. Appl. Vol. 94, pp. 105-134.

[3] Brosamler G. (1988). An almost everywhere central limit theorem. Math. Proc. Cambridge Phil. Soc. Vol. 104, pp. 561-574.

[4] Fazekas I., Rychlik Z. (2003). Almost sure central limit theorems for random filds. Math. Nachr. Vol. 259, pp. 12-18.

[5] Ibragimov I.A. (1996). On almost sure versions of limit theorems. (In Russian.) Dokl. Acad. Nauk. Vol. 350, pp. 301-303.

[6] Kruglov V.M., Petrovskaya G.N. (2002). Weak convergence of self-normalized random poligonal lines. Journal of Mathematical Sciences. Vol. 112, pp. 4145-4154.

[7] Rényi A. (1953). On the the theory of order statistics. Acts Math. Acad. Sci. Hung. Vol. 4, pp. 191-231.

[8] Schatte P. (1988). On strong versions of the central limit theorem. Math. Nachr. Vol. 137, pp. 249-256. 